\section{A Switched LQ Regulator Design in Continuous Time}

\author{
Pierre Riedinger
}

\begin{abstract}
In this technical note, the design of a $L Q$ regulator for linear switched systems in continuous time is investigated. From a relaxation of the optimal control problem, a Lyapunov based switching law is provided. Even if the subsystems are all unstable, the state feedback switching law can be applied subject to a positiveness condition. In any cases, the real cost is always upper bounded by the Lyapunov function value. The optimality of the switching law is also discussed and we prove that the switching conditions are optimal in some generic cases. This point explains why the obtained results over examples approach finely the optimal solutions. Finally, a design strategy is also given that extends the results to the cases where the subsystems are controlled linear systems.
\end{abstract}

Index Terms - LQ regulator, optimal control, switched systems.

\section{INTRODUCTION}

In the last decade, many contributions have addressed the design of stabilizing switching laws for switched systems both in continuous and discrete time (see for examples [1]-[4] for dynamic programming approaches, [5]-[7] for variational approaches, and [8]-[10] for Lyapunov based approaches). This problem cannot be considered trivial even numerically [7], [11] and the goal to design closed loop control, based on the optimization of a criteria, is a challenging task.

LQ regulators play a central role in the control theory of linear systems due to their simple design to meet performance requirements and their robustness properties. Up to now, the exact solution of a switched LQ problem is not available. Only approximations of the state feedback switching law have been proposed in the literature using the dynamic programming [1]-[4]. The practical obstacle to the application of these methods relies on the difficulties to compute numerically a good approximation of the solution not only for small dimensional problems. Alternatively, open loop control can be achieved using direct or indirect optimization methods [6], [7] but singular solutions [5], [12] entail numerical difficulties [11].

In [10], Lyapunov based approaches are developed for a mode independent quadratic cost and the authors show that the design conditions are not convex and the problem cannot be solved by Linear Matrix Inequality (LMI) tools. A switched quadratic Lyapunov approach is also proposed in [8], based on Lyapunov-Metzler inequalities. If some LMI conditions can be guaranteed, the method allows to define a state feedback switching law and can be applied even when subsystems are unstable. An upper bound on the cost is also provided but the optimality of the proposed stabilizing switched law is not evaluated.

In this technical note, a variational approach has been used to analyze the optimal necessary conditions occurring in the switched LQ

Manuscript received January 17, 2013; revised May 15, 2013; accepted October 17, 2013. Date of publication November 06, 2013; date of current version April 18, 2014. This work was supported by the European Community's Seventh Framework Programme (FP7/2007-2013) under grant agreement n ${ }^{\circ} 257462$ and by HYCON2 Network of Excellence "Highly-Complex and Networked Control Systems". Recommended by Associate Editor H.L. Trentelman.

The author is with the Universite de Lorraine, CNRS-CRAN UMR 7039, Vandoeuvre-lès-Nancy 54516, France (e-mail: pierre.riedinger@univ-lorraine. fr).

Color versions of one or more of the figures in this technical note are available online at http://ieeexplore.ieee.org.

Digital Object Identifier 10.1109/TAC.2013.2289032 problem. A first result yields the right expression of the optimal cost with respect to the state and the co-state. From this analysis, a control Lyapunov function as well as a state feedback switching law is determined.

An important point which is discussed, is that the obtained switching conditions are actually optimal in some generic cases when the optimal control is constant. This explains why the state feedback switching law leads to some appealing results close to the optimal. In any case, the value of Lyapunov function defines an upper bound on the real cost and gives a guarantee on performances.

These results are established in a relaxed framework for which the singular arcs [5], [12] that appear in the optimal solution, are properly taken into account. This is a key point since for this class of systems, the optimal solutions are frequently singular as indicating by the big quantity of randomly tested examples.

The technical note is organized as follows. Section II is dedicated to the problem formulation and to the necessary conditions that must be fulfilled to solve the optimal control problem. A preliminary result establishes the right expression of the optimal cost when the optimal switching law is said regular. In Section III, the main result of this technical note is given. A control Lyapunov function and an ad hoc switching law are determined. In Section IV, we discuss the optimality of the proposed switching law. In Section V. several examples illustrate that the proposed switching law is effectively optimal. We propose also a design strategy in the case where the subsystems are, eventually all non stabilizable, controlled linear systems.

\section{PRoblem Formulation AND PRELIminary Results}

We consider the class of linear switched systems in continuous time

$$
\dot{x}(t)=A_{\sigma(t)} x(t) \quad x(0)=x_{0}
$$

where $\sigma:[0,+\infty) \rightarrow S=\{1, \cdots, s\}$ denotes the switching law that selects the active mode at time $t$ by choosing among a finite collection of matrices $A_{i} \in \mathbb{R}^{n \times n}, i \in S$. Our aim is to design a state feedback switching law (i.e., $x \mapsto \sigma(x)$ ) for system (1) that approaches the optimal solution of the following optimization problem:

Problem 1: Minimize the switched quadratic criterion

$$
\min _{\sigma(\cdot)} \frac{1}{2} \int_{0}^{\infty} x^{T}(t) Q_{\sigma(t)} x(t) d t
$$

where $Q_{i}=Q_{i}^{T}>0, i \in S$ subject to $\dot{x}(t)=A_{\sigma(t)} x(t), x(0)=x_{0}$.

A usual framework [5], [11] to solve optimal control problem for switched systems ( $\left.\dot{x}=f_{i}(x), i \in S\right)$ is to solve its relaxed version, replacing the vector field set $\left(f_{i}(x)\right)$ by its convex hull $\left(\dot{x}=\operatorname{co}\left\{f_{i}(x)\right\}\right)$. At least, three reasons justify the convexification of the problem: (i) the solutions are well defined (Fillipov; [13]); (ii) the density of the switched system trajectories into the trajectories of its relaxed version [14]; (iii) the existence of singular optimal solutions are taking into account [5], [12].

The relaxed version of Problem 1 is then given as a pure continuous time optimal control problem consisting of replacing the matrices $Q_{i}$ and $A_{i}$ respectively by their convex combination i.e., $Q(\lambda(t))=$ $\sum_{i=1}^{s} \lambda_{i}(t) Q_{i}$ and $A(\lambda(t))=\sum_{i=1}^{s} \lambda_{i}(t) A_{i}$ where $\lambda(t) \in \Lambda=$ $\left\{\lambda \in \mathbb{R}^{s}: \sum_{i=1}^{s} \lambda_{i}=1, \quad \lambda_{i} \geq 0\right\}$ plays the role of the control variable. 
To apply Pontryagin Maximum Principle (PMP) for Problem 1 or its relaxed version, the Hamiltonian function is defined as follows:

$$
\mathcal{H}(x, \lambda, p)=\sum_{i=1}^{s} \lambda_{i} \mathcal{H}_{i}(x, p)
$$

with $\mathcal{H}_{i}(x, p)=p^{T} A_{i} x+\frac{1}{2} x^{T} Q_{i} x$ and where $p$ defines the co-state. This leads to the following classical necessary conditions for optimality [15]:

Theorem 1: Suppose that $\lambda^{*}$ is optimal with the corresponding state $x^{*}$. Then, there exists an absolutely continuous function $p^{*}$, named co-state, such that:

1) $p^{*} \not \equiv 0$;

2) $\dot{p}^{*}=\sum_{i=1}^{s} \lambda_{i}^{*}(t)\left(-A_{i}^{T} p^{*}-Q_{i} x^{*}\right)$ for almost all $t \in \mathbb{R}^{+}$;

3) $\lambda^{*}(t)=\arg \min _{\lambda \in \Lambda} \mathcal{H}\left(x^{*}(t), \lambda, p^{*}(t)\right)$;

4) $\mathcal{H}\left(x^{*}(t), \lambda^{*}(t), p^{*}(t)\right)=0$.

From Item 3 and (3), it is clear that if there exists $i \in S$ at time $t$ such that $\mathcal{H}_{i}\left(x^{*}(t), p^{*}(t)\right)<\mathcal{H}_{j}\left(x^{*}(t), p^{*}(t)\right), \forall j \in S \backslash\{i\}$, then the optimal control has to satisfy $\lambda_{i}^{*}(t)=1$ and $\lambda_{j}^{*}(t)=0, \forall j \in S \backslash\{i\}$.

A switching instant can occur at time $t$ if there exists at least a pair $(i, j) \in S^{2}$ such that $\mathcal{H}_{i}=\mathcal{H}_{j}=0$. At this time, the value of $\lambda$ cannot be determined directly. Actually, if we suppose that $0=\mathcal{H}_{i}=$ $\mathcal{H}_{j}<\mathcal{H}_{k}, \forall k \in S \backslash\{i, j\}$ then the values that satisfy the relation, $\lambda_{i}+\lambda_{j}=1$, are potential candidate for optimality. Moreover, a so called singular control $\lambda$ can exist, for which $0=\mathcal{H}_{i}=\mathcal{H}_{j}<\mathcal{H}_{k}$, $\forall k \in S \backslash\{i, j\}$ on a non empty time interval $(a, b)$. This is a well known situation in the literature [16]-[18] and second order necessary conditions given by the Generalized Legendre-Clebsh Condition [19], [20] are generally necessary to solve the optimal control problem.

Definition 1: Asingular control $\lambda($.$) is such that there exist at least$ two indices $i, j$, for which $\mathcal{H}_{i}=\mathcal{H}_{j}=0$ on a non zero measure time interval $(a, b)$ and satisfying $\lambda(t) \in \Lambda, \lambda_{k}(t) \neq 1 \forall k \in S, \forall t \in$ $(a, b)$. The corresponding part of the trajectory is named a singular arc.

Remark 1: A singular control defines a Fillipov solution [21] for the original switched system (1). Hence, it allows to extend properly the notion of optimal solution for switched systems. Roughly speaking when an optimal solution of the relaxed problem possesses singular arcs, these arcs define sliding surfaces for the switched system (1) which lead to chattering if the surface is attractive. It is noteworthy that only suboptimal solutions can be achieved for the switched systems due to the limited switching frequency; see for example [5].

Theorem 2: If $p^{*}(0)$ is an optimal value for the co-state at a given $x_{0}$, then $\eta p^{*}(0)$ is an optimal value for the initial state $\eta x_{0}$, for all $\eta \in \mathbb{R}$. Moreover, the optimal cost $V^{*}$ is homogenous of degree 2 : $V^{*}\left(\eta x_{0}\right)=\eta^{2} V^{*}\left(x_{0}\right)$.

Proof: First, observe by linearity that applying the same control $\lambda$ from two initial positions $x_{0}$ and $\eta x_{0}$ that the resulting trajectories are homogeneous of degree one i.e., $x\left(t, \eta x_{0}\right)=\eta x\left(t, x_{0}\right)$. It follows that the associated cost $V\left(x_{0}, \lambda\right):=\frac{1}{2} \int_{0}^{\infty} x^{T}(t) Q_{\lambda(t)} x(t) d t$ are also given by : $V\left(\eta x_{0}, \lambda().\right)=\eta^{2} V\left(x_{0}, \lambda().\right)$. In particular, it is obvious that if $\lambda^{*}$ is an optimal control for initial state $x_{0}$, then it is also an optimal one for the initial position $\eta x_{0}$ (easy to show by contradiction). It remains to show that if $p^{*}(0)$ is an optimal value for the co-state for a given initial position $x_{0}$ then $\eta p^{*}(0)$ is for $\eta x_{0}$. This is achieved if the two corresponding control laws are the same. Due to the homogeneity of $(x, p)$, the switching condition $\lambda^{*}(t)=$ $\arg \min _{\lambda \in \Lambda} \mathcal{H}\left(x^{*}(t), \lambda, p^{*}(t)\right)$ is not modified when we integrate the Hamiltonian system from $\left(x_{0}, p(0)\right)$ or from $\left(\eta x_{0}, \eta p(0)\right)$.

Remark 2: If the value function $V^{*}$ is homogeneous with degree two then its gradient is also homogeneous with degree one (at points where it can be defined).
Theorem 3: Assuming that Problem 1 admits a solution for each $x_{0}$, then the value function $V^{*}\left(x_{0}\right)$ is continuous.

Proof: It can be achieved using the fact that for given control $\lambda \in \Lambda$ and a given $R>0, A(\lambda) x$ is clearly Lipschitz on any ball $B(0, R)$ as well as $x^{T} Q(\lambda) x$. Then, an adapted proof and inspired by [[22, chap. 8 pp. 188-189]] can be applied to get the result.

Remark 3: Unfortunately, we fail to prove that $V^{*}$ is also Lipschitz and then differentiable almost everywhere (which is the case for the optimal control problem states in finite time).

Now, consider the set of Lyapunov equations corresponding to each subsystem $\left(A_{i}\right), i \in S$ for which a symmetric solution $P_{i}$ exists and is unique

$$
A_{i}^{T} P_{i}+P_{i} A_{i}+Q_{i}=0 .
$$

Let us define the variables $p_{i}$ from the co-state $p$ as

$$
p=p_{i}+P_{i} x, \forall i \in S
$$

then, the Hamiltonian function $\mathcal{H}$ can be simplified to $\mathcal{H}=$ $\sum_{i=1}^{s} \lambda_{i} \mathcal{H}_{i}$ with $\mathcal{H}_{i}=p_{i}^{T} A_{i} x$. It is straightforward to verify that when subsystem $i$ is active on a time interval $(a, b)$, i.e., $\forall t \in(a, b)$, $\lambda_{i}(t)=1$ and $\lambda_{j}(t)=0, \forall j \in S \backslash\{i\}$, the dynamic of $p_{i}$ is given by

$$
\dot{p}_{i}=-A_{i}^{T} p_{i}
$$

while the evolutions of the others $p_{j}$ for all $j \in S \backslash\{i\}$ follow from (5):

$$
p_{j}=p_{i}+\left(P_{i}-P_{j}\right) x .
$$

Item 4 in above Theorem 1 yields

$$
\mathcal{H}_{i}=p_{i}^{T} A_{i} x \equiv 0, \quad \text { on }(a, b)
$$

and a switch can only occur from subsystem $\left(A_{i}\right)$ to subsystem $\left(A_{j}\right)$ at time $t=b$, if

$$
\mathcal{H}_{i}=\mathcal{H}_{j} \Leftrightarrow p_{i}^{T} A_{i} x=p_{j}^{T} A_{j} x=0
$$

and before this switch, the following conditions are fulfilled:

$$
\mathcal{H}_{j}=p_{j}^{T} A_{j} x>0 \text {, for all } j \in S \backslash\{i\} .
$$

Theorem 4: Assume there exists a non singular stabilizing control law $\lambda$ and a co-state $p$ such that Theorem 1 is satisfied. Denote by $\left\{t_{0}, t_{1}, t_{2}, \cdots\right\}$ with $t_{0}=0$, the associated switching time sequence (possibly infinite) and by $\left\{i_{0}, i_{1}, i_{2}, \cdots\right\}$ the corresponding mode sequence. Then, the cost function is determined by

$$
V\left(x_{0}, \lambda\right)=\frac{1}{2}\left(x_{0}^{T} P_{i_{0}} x_{0}+p_{i_{0}}(0)^{T} x_{0}-L\right)
$$

where $L=\lim _{k \rightarrow \mathcal{N}} x_{k}^{T} p_{i_{k}}\left(t_{k}\right)$ with $x_{k}:=x\left(t_{k}\right)$ and $\mathcal{N} \in$ $\mathbb{N} \bigcup\{+\infty\}$ the number of switchings. If the trajectory is optimal, $L=0$ and the co-state $p_{i_{0}}(t)$ for $t_{0} \leq t \leq t_{1}$ can be identified with

$$
p_{i_{0}}(t)=\sum_{k=1}^{\mathcal{N}} \Pi^{T}\left(t, t_{k}\right)\left(P_{i_{k}}-P_{i_{k-1}}\right) x_{k}
$$

where $\Pi\left(t, t_{k}\right)$ is the transition function from time $t$ to $t_{k}$. 
Proof: As it is assumed that the switching sequence is non singular, the cost function can be written

$$
V\left(x_{0}, \lambda\right)=\frac{1}{2} \int_{0}^{t_{1}} x^{T} Q_{i_{0}} x d t+\frac{1}{2} \int_{t_{1}}^{t_{2}} x^{T} Q_{i_{1}} x d t+\cdots
$$

Using (4) and integrating, we get

$$
V\left(x_{0}, \lambda\right)=\frac{1}{2} x_{0}^{T} P_{i_{0}} x_{0}+\frac{1}{2} \sum_{k=1}^{\mathcal{N}} x_{k}^{T}\left(P_{i_{k}}-P_{i_{k-1}}\right) x_{k}
$$

where $x_{k}:=x\left(t_{k}\right)$ is the state at switching times $t_{k}, k=\{0, \ldots, \mathcal{N}\}$.

Define $G:=\sum_{k=1}^{\mathcal{N}} x_{k}^{T}\left(P_{i_{k}}-P_{i_{k-1}}\right) x_{k}$. Using (7), $G$ can be written as

$$
\begin{aligned}
G & =\sum_{k=1}^{\mathcal{N}} x_{k}^{T}\left(p_{i_{k-1}}-p_{i_{k}}\right)\left(t_{k}\right) \\
& =x_{1}^{T} p_{i_{0}}\left(t_{1}\right)-x_{1}^{T} p_{i_{1}}\left(t_{1}\right)+x_{2}^{T} p_{i_{1}}\left(t_{2}\right)+\cdots \\
& -x_{\mathcal{N}-1}^{T} p_{i_{\mathcal{N}-1}}\left(t_{\mathcal{N}-1}\right)+x_{\mathcal{N}}^{T} p_{i_{\mathcal{N}-1}}\left(t_{\mathcal{N}}\right)-x_{\mathcal{N}}^{T} p_{i_{\mathcal{N}}}\left(t_{\mathcal{N}}\right) \\
& =x_{1}^{T} p_{i_{0}}\left(t_{1}\right)-x_{\mathcal{N}}^{T} p_{i_{\mathcal{N}}}\left(t_{\mathcal{N}}\right) .
\end{aligned}
$$

The last equality is due to the fact that the product $x^{T}(t) p_{i_{k}}(t)$ is constant on time interval $\left(t_{k}, t_{k+1}\right)$ which implies that all the differences disappear. Denoting $L$ by $L=x_{\mathcal{N}}^{T} p_{i_{\mathcal{N}}}\left(t_{\mathcal{N}}\right)$ establishes the cost expression (11).

Now, we assume that the switching sequence is optimal. If $\mathcal{N}$ is finite then it means a last mode is used. Consequently, as for a classical LQ problem, the co-state after the last switch equals to $p=P_{\mathcal{N}} x$ and it follows that $p_{i_{\mathcal{N}}}=0$ and $L=0$.

If $\mathcal{N}$ is infinite, the term $G$ defines a telescoping series which is convergent if and only if $x_{N}^{T} p_{i_{N}}\left(t_{N}\right)$ converges to a limit $L$. The value of the series is then $x_{1}^{T} p_{i_{0}}\left(t_{1}\right)-L$. As the control problem is homogenous (By homogenous, we means that if $p_{0}$ is the optimal co-state value corresponding to an initial condition $x_{0}$ then $\alpha p_{0}$ is for $\alpha x_{0}$ ), the only possible value for $L$ is zero when the extremal is optimal. Else it implies that $p$ goes to infinity when $x$ is steered to the origin which contradicts the homogeneity of the problem. This can be also viewed as follows: Considering the optimal control problem in finite time $t_{f}$, then the necessary condition $p\left(t_{f}\right)=0$ implies from (5) that $p_{i\left(t_{f}\right)}=-P_{i\left(t_{f}\right)} x\left(t_{f}\right)$. Then, taking the limit $\lim _{t_{f} \rightarrow+\infty} p_{i\left(t_{f}\right)}=\lim _{t_{f} \rightarrow+\infty}-P_{i\left(t_{f}\right)} x\left(t_{f}\right)=0$ since $\lim _{t_{f} \rightarrow+\infty} x\left(t_{f}\right)=0$ and $i\left(t_{f}\right) \in S$ is finite.

Using one more time the fact that the product $x^{T}(t) p_{i_{0}}(t)$ is constant between $\left(t_{0}, t_{1}\right)$, it can be concluded that the value function equals to

$$
V^{*}\left(x_{0}\right)=\frac{1}{2}\left(x_{0}^{T} P_{i_{0}} x_{0}+p_{i_{0}}\left(t_{0}\right)^{T} x_{0}\right) .
$$

Moreover, denote by $\Pi(a, b)$ the transition function from time $t=a$ to time $t=b$, then the cost function can be explicitly defined in terms of $x_{0}$ and of the switching time sequence $T=\left(t_{0}, t_{1}, t_{2}, \ldots\right)$ by

$$
\begin{aligned}
& V\left(x_{0}, T\right)= \\
& \frac{1}{2}\left(x_{0}^{T} P_{i_{0}} x_{0}+x_{0}^{T}\left(\sum_{k=1}^{\mathcal{N}} \Pi^{T}\left(t_{0}, t_{k}\right)\left(P_{i_{k}}-P_{i_{k-1}}\right) \Pi\left(t_{0}, t_{k}\right)\right) x_{0}\right)
\end{aligned}
$$

and $p_{i_{0}}(t)$ in (11) could be identified as in (12).

Remark 4: Equation (11) shows that the cost function not only depends on $x_{0}$ as in the standard LTI LQ Problem. Unfortunately, as we can see in (15), to obtain the value of the cost, the switching states $x_{k}$ (or the switching instants $t_{k}$ ) must be available.

Theorem 5: The variable $p_{i_{k}}$ defined by (5) in the time interval $\left(t_{k}, t_{k}+1\right)$ is given by $p_{i_{k}}(t)=\tilde{P}_{i_{k}}(t) x(t)$ where the time variant symmetric matrix $\tilde{P}_{i_{k}}(\cdot)$ follows the differential Lyapunov equation:

$$
\begin{aligned}
-\dot{\tilde{P}}_{i_{k}}(t) & =A_{i_{k}}^{T} \tilde{P}_{i_{k}}(t)+\tilde{P}_{i_{k}}(t) A_{i_{k}} \\
\tilde{P}_{i_{k}}\left(t_{k+1}\right) & =\tilde{P}_{i_{k+1}}\left(t_{k+1}\right)+P_{i_{k+1}}-P_{i_{k}} .
\end{aligned}
$$

Proof: The proof is obvious in view of (15) and from the fact that for $t_{k} \leq t \leq t_{k+1}, \tilde{P}_{i_{k}}(t)=e^{A_{i_{k}}^{T^{\prime}}\left(t_{k+1}-t\right)} \tilde{P}_{i_{k}}\left(t_{k+1}\right) e^{A_{i_{k}}\left(t_{k+1}-t\right)}$.

In view of these preliminary results, it appears that the state space is partitioned following the mode $i \in S$ which is used. Along an optimal trajectory, when mode $i_{k} \in S$ is used, the optimal cost is of the form $V^{*}(x)=x^{T} P_{i_{k}} x+c_{i_{k}}$ with $c_{i_{k}}=x^{T} \tilde{P}_{i_{k}} x$ a constant. At switching time i.e., on the boundary of the partition, the next constant is given by $c_{i_{k+1}}=c_{i_{k}}+x^{T}\left(P_{i_{k}}-P_{i_{k+1}}\right) x$. Unfortunately, as the given expression of the optimal cost is related to a trajectory (particularly the constants $c_{i_{k}}$ ), the right expression of the value function is not available on the whole state space and it is not possible to use directly Hamilton-Jacobi-Bellman equation to determine the state space partition. In the next section, a control Lyapunov function is exhibited that allows to approach finely the optimal solution in some generic cases as the Sections IV and V will show.

\section{LyAPUNOV BASED SWITCHING LAW}

The aim of this part is to define a Lyapunov function as a tight upper bound on the value function. We means tight in the sense that the two functions may coincide at some points.

Consider the set of Lyapunov equations corresponding to each subsystems $(A(\lambda)), \lambda \in \Lambda$ for which a symmetric solution $P_{\lambda}$ exists and is unique if the eigenvalues, $\alpha_{k}, k=1, \cdots, n$ of $A(\lambda)$, satisfy $\alpha_{i}+\alpha_{j} \neq 0$ for all pair $(i, j)$

$$
A(\lambda)^{T} P_{\lambda}+P_{\lambda} A(\lambda)+Q(\lambda)=0 .
$$

Definition 2: For a fixed $M>0$ (arbitrary large), let us define the set

$$
\Lambda^{+}(M)=\left\{\lambda \in \Lambda: P_{\lambda}>0, \max \left(\operatorname{eig}\left(P_{\lambda}\right)\right) \leq M\right\}
$$

where $\operatorname{eig}(P)$ stands for the set of eigenvalues of the matrix $P$.

The following proposition holds:

Proposition 1: If $Q_{i}>0$, for all $i \in S$ then $\Lambda^{+}(M)$ is a compact set and there exists a $\alpha_{\min }=\min _{\lambda \in \Lambda^{+}(M)} \min \left(\operatorname{eig}\left(P_{\lambda}\right)\right)>0$.

Proof: $\Lambda^{+}(M)$ is obviously bounded. Let us show it is a closed set. Suppose that there exists a sequence $\lambda_{k} \in \Lambda^{+}(M)$ such that $\min \left(e i g\left(P_{\lambda_{k}}\right)\right) \rightarrow 0$. As $\Lambda$ is a compact set, there exists a subsequence that converges to an element $\lambda \in \Lambda$ and such that $\min \left(e i g\left(P_{\lambda}\right)\right)=0$. Denote by $v_{0}$ the associated eigenvector. It follows the contradiction from $2 v_{0}^{T} P_{\lambda} A(\lambda) v_{0}+v_{0}^{T} Q(\lambda) v_{0}=v_{0}^{T} Q(\lambda) v_{0}=0$ which is absurd since $Q(\lambda)=\sum_{i \in S} \lambda_{i} Q_{i}>0$. Then, $\Lambda^{+}(M)$ is a closed set and there exists $\alpha_{\text {min }}>0$ such that $\forall \lambda \in \Lambda^{+}(M)$, $\min \left(\operatorname{eig}\left(P_{\lambda}\right)\right) \geq \alpha_{\text {min }}$.

It can now be introduced the following min-type function and proposition:

Proposition 2: The function defined by

$$
x \mapsto V_{\min }(x):=\min _{\lambda \in \Lambda^{+}(M)} x^{T} P_{\lambda} x
$$

is locally Lipschitz continuous and proper.

Proof: The solution of (18) are always well defined on $\Lambda^{+}(M)$ and vary continuously with $\lambda$. Then, by compacity and 
for any fixed $x$, the minimum exists and $\inf _{\lambda \in \Lambda+(M)} x^{T} P_{\lambda} x=$ $\min _{\lambda \in \Lambda+(M)} x^{T} P_{\lambda} x$.

Moreover, the familly of function indexed by $\lambda \in \Lambda^{+}(M)$ and defined by $x \mapsto x^{T} P_{\lambda} x$ is uniformly locally Liptchitz on any ball $\mathrm{B}(0, \mathrm{R})$ with a Lipschitz constant equals to $K:=2 M R$. This is obvious since for all $\lambda \in \Lambda^{+}(M)$

$$
\alpha_{\min }\|x\|^{2} \leq \inf _{\lambda \in \Lambda^{+}(M)} x^{T} P_{\lambda} x \leq x^{T} P_{\lambda} x \leq M\|x\|^{2} .
$$

So, as any $x^{T} P_{\lambda} x$ have a Lipschitz constant equals to $K$ on a ball of radius $R$, we can conclude that it is also the case for the minimum. Indeed, for any pair $(x, y)(\in B(0, R))$, there exists a pair $\left(\lambda_{1}, \lambda_{2}\right) \in$ $\Lambda^{+}(M)^{2}$ such that

$$
\left|V_{\min }(x)-V_{\min }(y)\right|=\left|x^{T} P_{\lambda_{1}} x-y^{T} P_{\lambda_{2}} y\right|
$$

Thus, by continuity, there exists $z$ on the line segment $\left[\begin{array}{ll}x & y\end{array}\right]$ such that $z^{T} P_{\lambda_{1}} z=z^{T} P_{\lambda_{2}} z$ and it follows:

$$
\begin{aligned}
\left|V_{\min }(x)-V_{\min }(y)\right| & \leq\left|x^{T} P_{\lambda_{1}} x-z^{T} P_{\lambda_{1}} z\right| \\
& +\left|z^{T} P_{\lambda_{2}} z-y^{T} P_{\lambda_{2}} y\right| \\
& \leq K\|x-z\|+K\|z-y\| \\
& =K\|x-y\| .
\end{aligned}
$$

The min type function $V_{\min }$ is also proper in view of the left part of inequality (21).

Definition 3: Let us define $L(x)$ as the set of values $\lambda \in \Lambda^{+}(M)$ where the minimum $V_{\min }(x)$ is achieved and $I(x)$ as the set of indices where the minimum over $i \in S$ of $x^{T}\left(P_{\lambda}(x)-P_{i}\right) A_{i} x, \lambda \in L(x)$, is reached

$$
I(x):=\arg \min _{i \in S, \lambda \in L(x)} x^{T}\left(P_{\lambda}-P_{i}\right) A_{i} x .
$$

The following lemma is useful latter in order to define an appropriate descent direction for $V_{\min }$ :

Lemma 1: For every $\lambda \in \Lambda$, such that (18) admits a solution, $\min _{i \in S} x^{T}\left(P_{\lambda}-P_{i}\right) A_{i} x$ is non positive.

Proof: For a given $\lambda \in \Lambda$, left-multiplying by $x^{T}$ and rightmultiplying by $x$ the (18) and using $Q(\lambda)=\sum_{i \in S} \lambda_{i} Q_{i}$ and $A_{i}^{T} P_{i}+$ $P_{i} A_{i}+Q_{i}=0$, we get

$$
\sum_{i \in S} \lambda_{i} x^{T}\left(P_{\lambda}-P_{i}\right) A_{i} x=0
$$

So, we cannot have $x^{T}\left(P_{\lambda}-P_{i}\right) A_{i} x>0$ for every $i \in S$ because in this case the left-hand member of equality (26) would be positive.

We can now state one of the main result of this technical note:

Theorem 6: Assume there exists at least a $\lambda \in \Lambda$ such that $A(\lambda)$ is Hurwitz. Then, the state feedback switching rule defined by

$$
i^{*}(x) \in I(x)=\arg \min _{i \in S, \lambda \in L(x)} x^{T}\left(P_{\lambda}-P_{i}\right) A_{i} x
$$

stabilizes the switching system (1) with a cost smaller than $\frac{1}{2} V_{\min }\left(x_{0}\right)$.

Proof: Let $\lambda \in \Lambda$ such that $A(\lambda)$ is Hurwitz, then the Lyapunov equation (18) admits a (unique) solution $P_{\lambda}>0$. The matrix $A\left(\lambda^{0}\right)$ is still Hurwitz if $\lambda^{0}$ belongs to a sufficiently small neighborhood of $\lambda$ and so (18) admits a unique solution for every $\lambda^{0}$ in some neighborhood of $\lambda$. Thus, the interior of $\Lambda^{+}$is non empty and $V_{\min }$ is well defined.
Let us now consider the directional derivative of $V_{\min }(x(t))$ with respect to $\dot{x}=A(\nu) x, \nu \in \Lambda$ as

$$
V_{\min }^{\prime}(x ; \nu)=\lim _{h \rightarrow 0 ; h>0} \frac{V_{\min }(x+h A(\nu) x)-V_{\min }(x)}{h} .
$$

Denote by $g_{\lambda}(x):=x^{T} P_{\lambda} x$ the continuous function defined on $\Lambda^{+}(M) \times \mathbb{R}^{n}$. Then, as the following conditions are met: 1 . the set $\Lambda^{+}(M)$ is compact, 2. for each $\lambda \in \Lambda^{+}(M)$, the directional derivative $g_{\lambda}^{\prime}(x, \cdot)$ exists, $3 . \forall w \in \mathbb{R}^{n}, \frac{g_{\lambda}(x+h w)-g_{\lambda}(x)}{h} \rightarrow g^{\prime}(x ; w)$ uniformly in $\lambda$ as $h \rightarrow 0$, it can be concluded (from Theorem 6.1, [23, p. 350-353])

$$
V_{\min }^{\prime}(x ; \nu)=\min _{\lambda \in L(x)} 2 x^{T} P_{\lambda} A(\nu) x .
$$

Taking $\nu=i^{*}(x) \in I(x)$, it follows using Lemma 1 that:

$$
\begin{aligned}
V_{\text {min }}^{\prime}\left(x, i^{*}(x)\right) & =\min _{\lambda \in L(x)} 2 x^{T} P_{\lambda} A_{i^{*}(x)} x \leq-x^{T} Q_{i^{*}(x)} x<0, \\
x & \neq 0 .
\end{aligned}
$$

Therefore, for any initial condition $x_{0}$

$$
V_{\min }(x(t))+\int_{0}^{t} x(\tau)^{T} Q_{i^{*}(x(\tau))} x(\tau) d \tau \leq V_{\min }\left(x_{0}\right), \quad \forall t \geq 0
$$

As $Q_{i}>0, \forall i \in S$ and as $V_{\min }(\cdot)$ is proper, it follows that: $V_{\min }(x(t)) \rightarrow 0$ and $x(t) \rightarrow 0$ when $t \rightarrow+\infty$.

\section{Discussion CONCERning THE SWITCHING LAW AND ITS OPTIMALITY}

In this part we want to discuss the degree of optimality of the provided switching law. Observe first that in the case where all matrices $A_{i}$ are Hurwitz, then the matrices $P_{i}$ are definite positive for each mode $i$ and the Lyapunov function $V_{\min }$ satisfies always the following inequality: $V_{\min }(x) \leq \min _{i \in S} x^{T} P_{i} x$. One can also observe that for a given initial state $x$, the value $\frac{1}{2} V_{\min }(x)$ is the best cost related to every constant convex combination that stabilizes the relaxed system. As we will see, this is an important point if a singular control occurs in the solution.

Now, in the general case, when can we say that $\frac{1}{2} V_{\min }(x)$ is optimal? The answer is :'Along the part of trajectories where the optimal control $\lambda^{*}$ is constant to reach the origin."

In order to prove this last sentence, consider an optimal solution and assume that the optimal control is piecewise smooth with a countable number of discontinuities occurring at time $t_{1}, t_{2}, \ldots$ Then, on time interval $\left(t_{k}, t_{k+1}\right)$, the optimal control $\lambda^{*}$ is smooth and we can denote by $\bar{A}(t)=A\left(\lambda^{*}(t)\right), \bar{Q}(t)=Q\left(\lambda^{*}(t)\right)$, the time varying matrices. The co-state $p(t)$ can be identified as for a time varying linear system to $\bar{P}(t) x(t)$ (as suggested by Theorem 5 ) where the time varying matrix $\bar{P}(t)$ satisfies the Lyapunov equation

$$
-\dot{\bar{P}}=\bar{A}^{T} \bar{P}+\bar{P} \bar{A}+\bar{Q} .
$$

As it is mandatory that the hamiltonian $\mathcal{H}=p^{T} \bar{A} x+\frac{1}{2} x^{T} \bar{Q} x$ remains equal to zero, it implies that along the optimal trajectory: $x^{T} \dot{\bar{P}} x=0$. Thus, $\mathcal{H}$ can be rewritten as in the case of (26) as follows:

$$
\mathcal{H}=\sum_{i \in S} \lambda_{i}^{*} x^{T}\left(\bar{P}-P_{i}\right) A_{i} x=0
$$




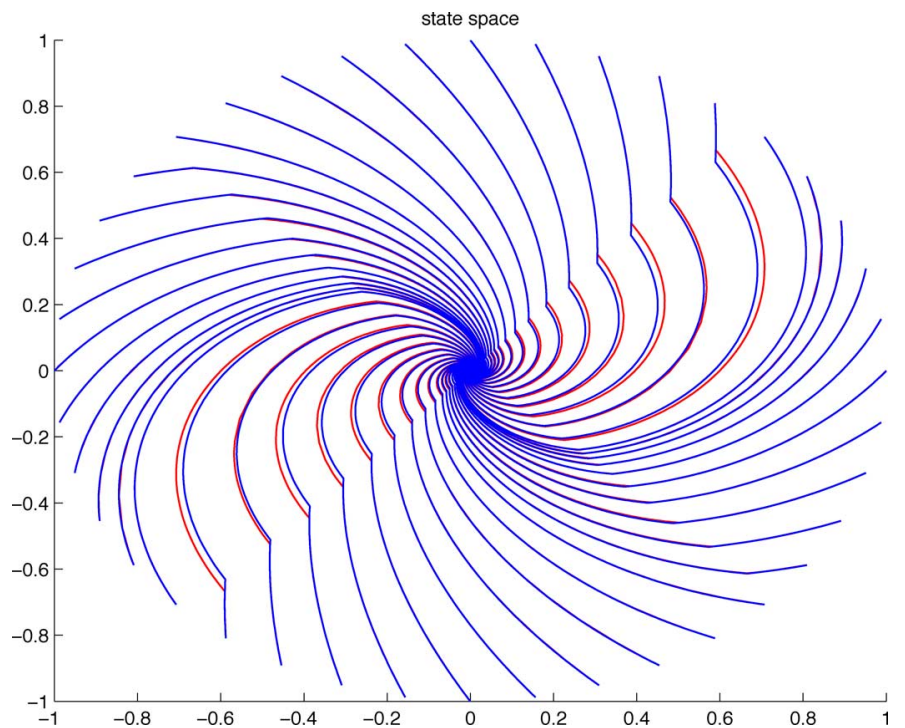

Fig. 1. Example 1: State space trajectories: (red) optimal solution (NLP); (blue) switching law.

where the optimal control $\lambda^{*}$ satisfies the complementarity constraint

$$
0 \leq \lambda_{i}^{*} \perp x^{T}\left(\bar{P}-P_{i}\right) A_{i} x \geq 0, i \in S .(x \perp y \text { means } x y=0) \text {. }
$$

Theorem 7: If after a given time instant $t$, the optimal control $\lambda^{*}$ stays constant on $(t,+\infty)$ and if $P_{\lambda^{*}}>0$ then the switching law provided by Theorem 6 coincides with the optimal one.

Proof: This is obvious since as in the classical LQ problem and after the last switch, the matrix $\bar{P}$ is given by the solution $P_{\lambda^{*}}$ of the Lyapunov equation (18) where $\lambda^{*}$ stands for the constant optimal control (eventually singular). Thus, as it is assumed that $P_{\lambda^{*}}>0$, by optimality, $x^{T} P_{\lambda^{*}} x=\min _{\lambda \in \Lambda: P_{\lambda}>0} x^{T} P_{\lambda} x$ and it can be concluded that $\lambda^{*} \in L(x)$. Along the optimal trajectory, the optimal switching condition is now based on the minimization of $x^{T}\left(P_{\lambda^{*}}-P_{i}\right) A_{i} x, i \in S$ which is exactly the switching condition provided by the switching rule of Theorem 6 . The resulting trajectories are then the same if $\lambda^{*}$ is admissible for the switched system. Else a chattering motion occurs around the sliding surface defined by conditions (32) and the trajectory can be considered to be similar of the optimal one provided that the switching frequency is sufficiently high [5].

Remark 5: Theorem 7 applies at least for two important generic cases: when the switching sequence is finite, and, in dimension 2 , along the singular arcs which are necessarily defined by lines passing through the origin and by a constant control [12].

Formally, we can justified the design of $V_{\min }$ as follow. Assuming known the value function, one can write for any $T>0, V^{*}\left(x_{0}\right)=$ $\min _{\sigma} \frac{1}{2} \int_{0}^{T} x^{T}(t) Q_{\sigma(t)} x(t) d t+V^{*}(x(T))$. The transversality condition of PMP implies at time $T, p^{*}(T)=\frac{\partial V(x(T))}{\partial x}$ (if exists). Now suppose that $V^{*}(x(T))$ is approximated by $V_{\min }(x(T))$. Then, an approximation of $p^{*}(T)$ is given by $p^{*}(x(T)) \approx P_{\lambda} x(T)$ with $\lambda \in L(x)$. Thus, it is easy to check that the minimization of the Hamiltonian at time $T$ leads to the switching law (27). As the problem is homogenous and if the approximation is "good", one can infer that $p^{*}(x) \approx P_{\lambda(x)} x$ with $\lambda(x) \in L(x)$ for every $x$. Roughly speaking, the switching law (27) matches the optimal one when $P_{\lambda(x)} x$ is a good approximation of $p^{*}$.

\section{ILLUSTRATIVE EXAMPLES}

Before presenting some examples, it is important to mention that it is not necessary to ensure a stabilizing switched law to determine all

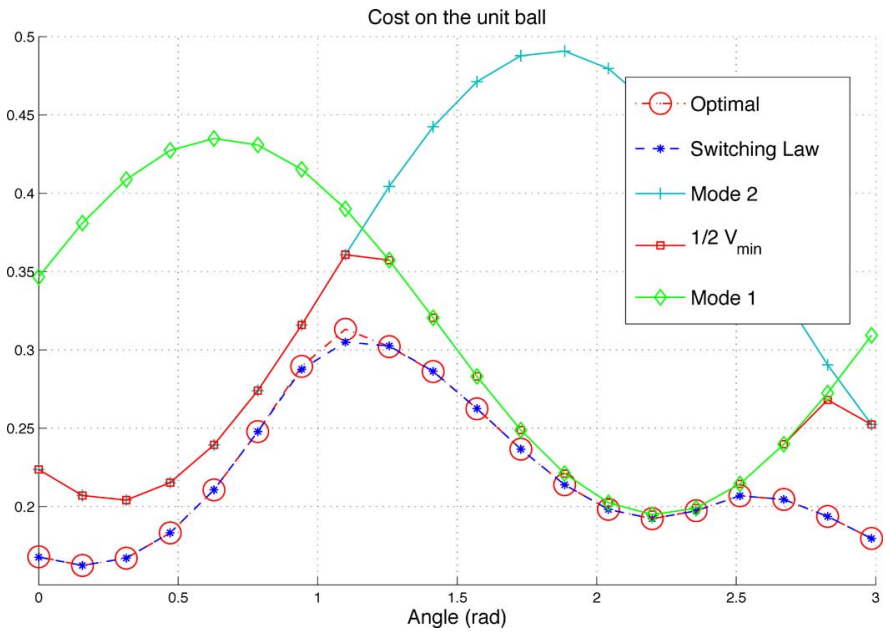

Fig. 2. Example 1: Cost comparisons for different initial positions taken on the unit ball.

the possible values of the set $\Lambda^{+}(M)$. Only one value is sufficient to guarantee the stability. So, a reasonable finite number of values ensures performances. A possibility to get this finite number is to discretize the set $\Lambda$.

\section{A. Example 1:A Regular Case}

In [24], we have proposed a periodic solution that approximated the optimal one for a switched system defined by two modes. The matrices corresponding to this example are

$$
\begin{aligned}
A_{1} & =\left(\begin{array}{cc}
-1.89 & 4.29 \\
-2.41 & -1.77
\end{array}\right), A_{2}=\left(\begin{array}{cc}
-1.14 & 0.95 \\
-1.23 & -1.57
\end{array}\right), \\
Q_{1} & =\left(\begin{array}{cc}
0.99 & -0.18 \\
-0.18 & 4.59
\end{array}\right), Q_{2}=\left(\begin{array}{cc}
2.15 & 0.66 \\
0.66 & 1.33
\end{array}\right) .
\end{aligned}
$$

For this example, there is no singular arc and the solution is always regular (and, thus, admissible for the switched system). Fig. 1 shows the state space trajectories for the switching law given by (27) and the optimal one. The later is obtained by NL programming in a suitable formulation taking into account singular arcs [11]. If not, numerical difficulties in the control determination are often encountered. We can see that the two solutions match well together. Thus, even if no argument has been advanced in this case, it seems that the provided switching conditions are close to the optimal.

Fig. 2 compares the optimal cost with the costs obtained by using the switching law, only mode 1 and only mode 2 , respectively. This comparison is made for initial states taken on the unit ball, the x-axis represent the angle $\theta$. It can be observed that the cost associated to the switching law coincides the cost of the optimal numerical solution. Of course, the essential difference is that the numerical solution is an open loop control while the switching law defines a closed loop control.

\section{B. Example 2:A Singular Case}

Let us take an example (chosen randomly) for which singular arcs occur in the solutions. The matrices for this example are

$$
\begin{aligned}
& A_{1}=\left(\begin{array}{cc}
-0.96 & -9.93 \\
0.68 & -5.08
\end{array}\right), A_{2}=\left(\begin{array}{cc}
-1.10 & 0.95 \\
1.88 & -2.76
\end{array}\right) \\
& Q_{1}=\left(\begin{array}{cc}
1.17 & 0.90 \\
0.90 & 5.60
\end{array}\right), Q_{2}=\left(\begin{array}{cc}
2.11 & -1.07 \\
-1.07 & 2.04
\end{array}\right) .
\end{aligned}
$$

As shown in Figs. 3 and 4, the result is clearly near optimal. By comparison, a min switching strategy defined by: $i(x)=$ 


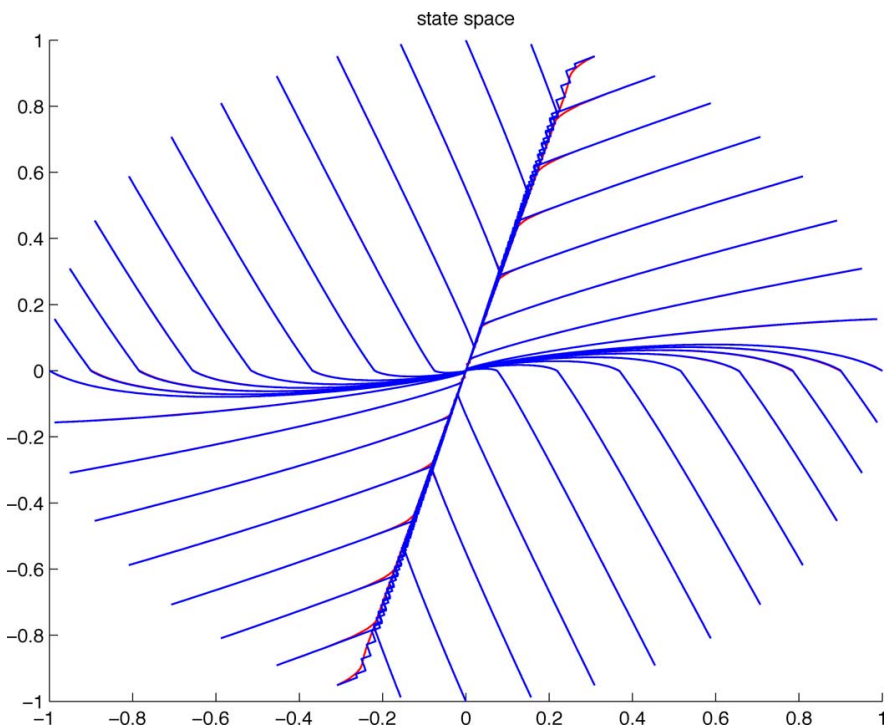

Fig. 3. Example 2 : State space trajectories: (red) optimal (NLP); (blue) switching law.

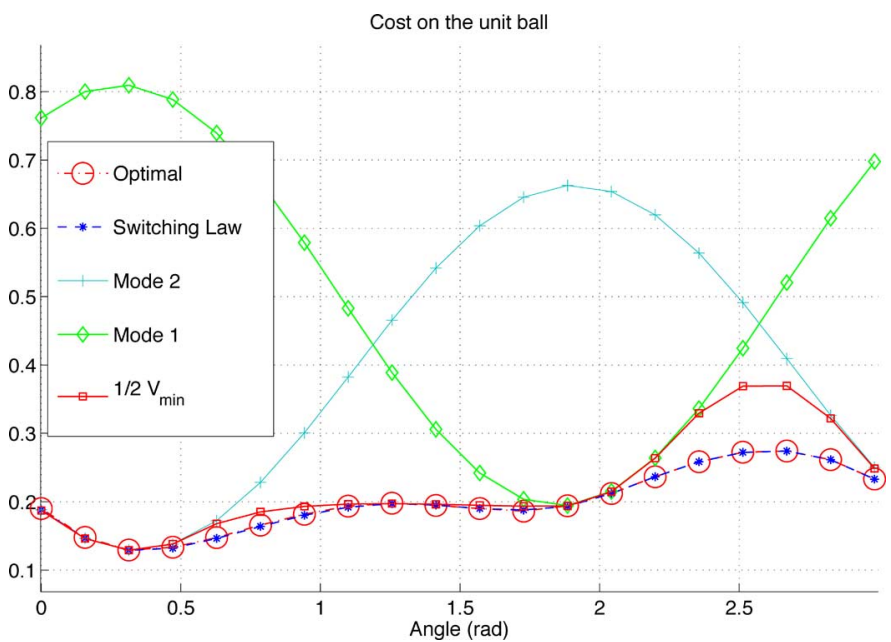

Fig. 4. Example 2: Cost comparisons for different initial positions taken on the unit ball.

$\arg \min _{i \in S}\left\{x^{T} P_{i} x\right\}$ leads to a cost given by $\frac{1}{2} \min _{i \in S}\left\{x^{T} P_{i} x\right\}$ which is clearly not optimal as the two examples show.

\section{Example 3 : Extension to Controlled Linear Switched Systems}

Consider the controlled linear switched systems in continuous time

$$
\dot{x}(t)=A_{\sigma(t)} x(t)+B_{\sigma(t)} u_{\sigma(t)}(t), \quad x(0)=x_{0}
$$

where $\sigma:[0,+\infty) \rightarrow S=\{1, \cdots, s\}$ denotes the switching law that selects the active mode at time $t$ by choosing among a finite collection of controlled linear systems. For $i \in S, A_{i} \in \mathbb{R}^{n \times n}, B_{i} \in \mathbb{R}^{n \times m_{i}}$, $x\left(\in \mathbb{R}^{n}\right)$ is the state and $u_{i}(t) \in \mathbb{R}^{m_{i}}\left(m_{i} \leq n\right)$ is the control associated to mode $i$.

We assume that the switched system is stabilizable but eventually all the pair $\left(A_{i}, B_{i}\right), i \in S$ cannot be stabilized.

A direct extension of the proposed switching law consists to solve:

Problem 2: Minimize

$$
\min _{\sigma(\cdot)} \frac{1}{2} \int_{0}^{\infty} x^{T}(t) \tilde{Q}_{\sigma(t)} x(t) d t
$$

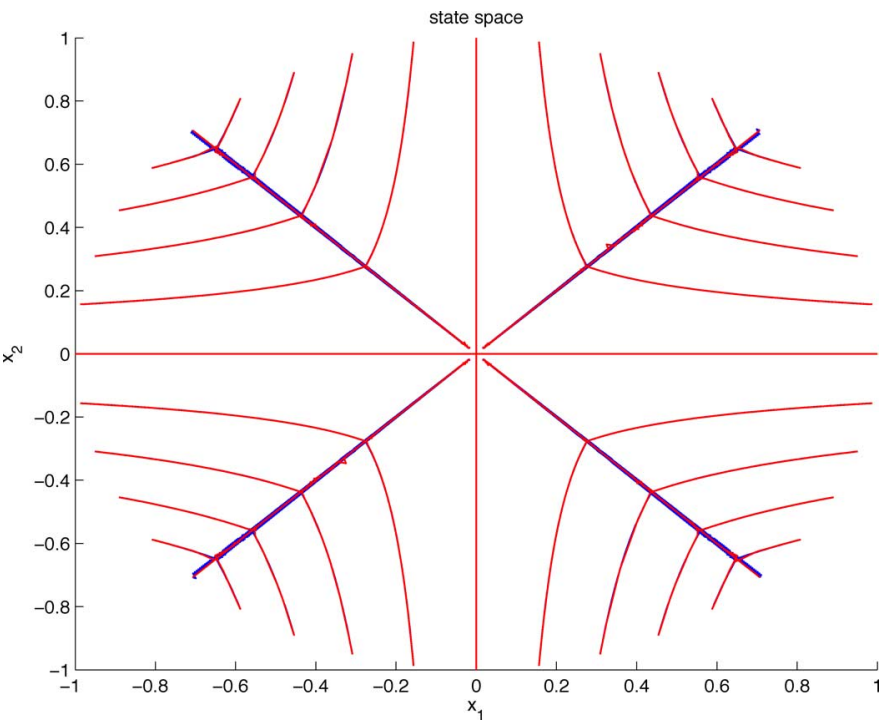

Fig. 5. Example 3 : State space trajectories: (red) optimal (NLP); (blue) switching law.

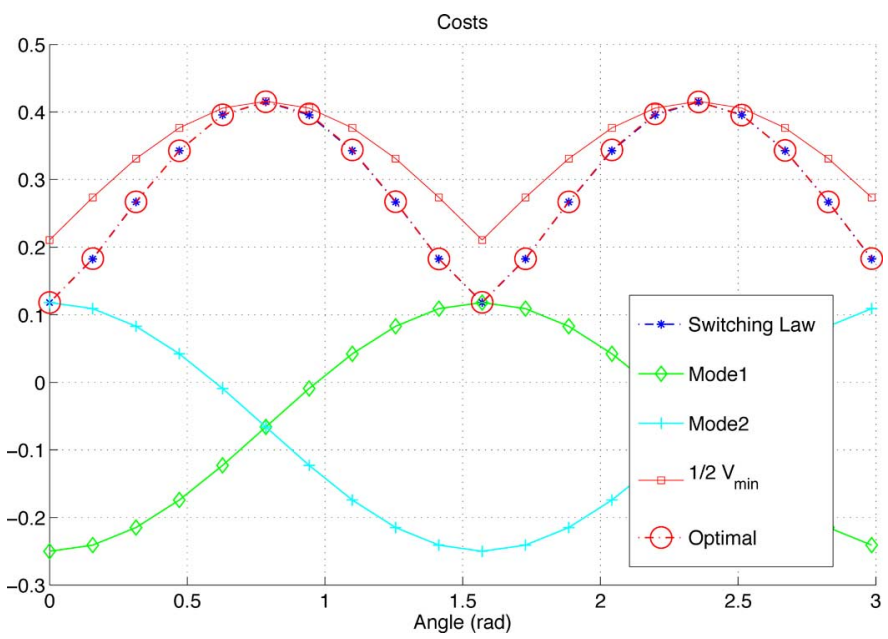

Fig. 6. Example 3: Cost comparisons for different initial positions taken on the unit ball.

subject to $\dot{x}(t)=\left(A_{\sigma(t)}-B_{\sigma(t)} K_{\sigma(t)}\right) x(t)$, where $\tilde{Q}_{i}=Q_{i}+$ $K_{i}^{T} R_{i} K_{i}$ and where matrices $\left(R_{i}, Q_{i}\right)$ and $K_{i}$ correspond to an LQ design for the subsystem $\left(A_{i}, B_{i}\right)$, for all $i \in S$.

Then, a stabilizing switching law can be obtained in two steps: 1.) Define static gain $K_{i}$ that stabilize the controllable subspace of each subsystems using a LQ design, 2.) Check if the switching law can be applied for Problem 2 i.e., if $\Lambda^{+}(M)$ is non empty for a sufficiently large $M$.

Let us illustrate our purpose on an example. Consider two non stabilizable subsystems defined by

$$
A_{2}=\left(\begin{array}{cc}
1 & 0 \\
0 & -2
\end{array}\right), A_{1}=\left(\begin{array}{cc}
-2 & 0 \\
0 & 1
\end{array}\right), B_{2}=\left(\begin{array}{l}
0 \\
1
\end{array}\right), B_{1}=\left(\begin{array}{l}
1 \\
0
\end{array}\right) .
$$

Clearly the controllable subspace is generated by the canonical basis vector $\left\{e_{1}=(1,0)^{T}\right\}$ for the first system while it is $\left\{e_{2}=(0,1)^{T}\right\}$ for the second. Thus, following the first step, an LQ design is used on each controllable subspace to determine two static gains $K_{1}$ and $K_{2}$ such that $\int_{0}^{\infty} x^{T} Q_{i} x+u_{i}^{T} R_{i} u_{i}$ is minimized by $u_{i}=-K_{i} x$. We have chosen $R_{i}=Q_{i}=1, i=1,2$ then $K_{1}=K_{2}=0.2361$. Thus, the switched linear system is now defined by: $\dot{x}=\left(A_{i}-B_{i} K_{i}\right) x$, $i=1,2$. 
The second step consists in applying the switching law with weight matrices $\tilde{Q}_{i}$. For this example, we have chosen : $\tilde{Q}_{i}=I d+K_{i}^{T} R_{i} K_{i}$, $i=1,2$. The Figs. 5 and 6 show once again that the obtained switching law is optimal by comparison with the numerical solution. It can be noticed that the solutions of (18) for mode 1 and 2 are not positive definite as expected, see Fig. 6.

\section{CONCLUSION}

A state feedback switching law based on control Lyapunov function for switched LQ regulator problems in continuous time has been proposed. The stabilizing feedback can be applied even if the subsystems are all unstable. The only condition that is required, is the existence of at least a stable convex combination of the subsystems. Even if the exact optimal solution is not determined, we have shown that the switching conditions involved by the switching law can be optimal. More precisely, this generic situation occurs along arcs (singular or not) ending to the origin with a constant optimal control. As in dimension two, the singular controls are constant, the switching law is really optimal in most of encountered 2-D examples. In any case, a guarantee on the cost is provided by an upper bound given by the value of the Lyapunov function.

\section{REFERENCES}

[1] X. Xu and P. J. Antsaklis, "Optimal control of switched systems based on parameterization of the switching instants," IEEE Trans. Autom. Control, vol. 49, no. 1, pp. 2-16, Jan. 2004.

[2] B. Lincoln and A. Rantzer, "Relaxing dynamic programming," IEEE Trans. Autom. Control, vol. 51, no. 8, pp. 1249-1260, 2006

[3] C. Seatzu, D. Corona, A. Giua, and A. Bemporad, "Optimal control of continuous-time switched affine systems," IEEE Trans. Autom. Control, vol. 51, no. 5, pp. 726-741, 2006.

[4] W. Zhang, J. Hu, and A. Abate, "On the value functions of the discretetime switched lqr problem," IEEE Trans. Autom. Control, vol. 54, no. 11, pp. 2669-2674, 2009.

[5] S. C. Bengea and R. A. DeCarlo, "Optimal control of switching systems," Automatica, vol. 41, no. 1, pp. 11-27, 2005.

[6] H. Axelsson, Y. Wardi, M. Egerstedt, and E. Verriest, "Gradient descent approach to optimal mode scheduling in hybrid dynamical systems," J. Optim. Theory Appl., vol. 136, no. 2, pp. 167-186, 2008.

[7] M. S. Shaikh and P. E. Caines, "On the hybrid optimal control problem: Theory and algorithms," IEEE Trans. Autom. Control, vol. 52, no. 9 , pp. 1587-1603, Sep. 2007.
[8] G. S. Deaecto, J. C. Geromel, and J. Daafouz, "Switched state feedback control for continuous time-varying polytopic systems," Int. J. Control., vol. 84, no. 9, pp. 1500-1508, 2011.

[9] P. Colaneri, J. C. Geromel, and A. Astolfi, "Stabilization of continuoustime switched nonlinear systems," Syst. Control Lett., vol. 57, no. 1, pp. 95-103, 2008.

[10] J. C. Geromel and P. Colaneri, "Stability and stabilization of discrete time switched systems," Int. J. Control, vol. 79, pp. 719-728, 2006.

[11] P. Riedinger and I. C. Morarescu, "A numerical framework for optimal control of switched input affine nonlinear systems subject to path constraint," Math. Comp. Simul., to be published.

[12] D. Patino, P. Riedinger, and C. Iung, "Practical optimal state feedback control law for continuous-time switched affine systems with cyclic steady state," Int. J. Control, vol. 82, no. 7, pp. 1357-1376, 2009.

[13] A. Filippov, Differential Equations with Discontinuous Righthand Sides. New York: Springer, 1988, vol. 18, Mathematics and its Applications (Soviet Series).

[14] B. Ingalls, E. D. Sontag, and Y. Wang, "An infinite-time relaxation theorem for differential inclusions," in Proc. Amer. Math. Soc., 2003 , vol. 131(2), pp. 487-499.

[15] L. S. Pontryagin, V. G. Boltyanskii, R. V. Gamkrelidze, and E. F. Mishchenko, The Mathematical Theory of Optimal Processes. Oxford, U.K.: Pergamon, 1964.

[16] H. M. Robbins, "A generalized Legendre-Clebsch-condition for the singular cases of optimal control," IBM J. Res. Develop., vol. 11, no. 4, pp. 361-372, 1967

[17] A. E. Bryson and Y.-C. Ho, Appl. Optimal Control. London, U.K.: Taylor \& Francis, 1975.

[18] B. Bonnard, C. Bernard, and M. Chyba, Singular Trajectories and their Role in Control Theory. New York: Springer SMAI, 2003, Series: Mathématiques et Applications.

[19] V. Michel, Singular Optimal Control - The State of the Art. Technical Report Univ. Kaiserslautern, Kaiserslautern, Germany, Tech. Rep., 1996.

[20] A. J. Krener, "The higher order maximal principle and its application to singular extremals," SIAM J. Control Optim., vol. 15, no. 2, pp. 256-293, 1977.

[21] J. Cortes, "Discontinuous dynamical systems," IEEE Control Syst. Mag., vol. 4, pp. 36-73, Jun. 2008.

[22] A. Bressan and B. Piccoli, Introduction to the Mathematical Theory of Control. Minneapolis, MN: American Institut of Mathematical Sciences, 2007.

[23] N. Furukawa, "Optimality conditions in nondifferentiable programming and their applications to best approximations," Appl. Math Optim., vol. 9, pp. 337-371, 1983.

[24] P. Riedinger, F. Kratz, C. Iung, and C. Zanne, "Linear quadratic optimization for hybrid systems," in Proc. IEEE Conf. Decision Control, 1999, pp. 3059-3064. 